\title{
Cutaneous Lupus Erythematosus Presenting as Frontal Fibrosing Alopecia: Report of 2 Patients
}

\author{
Ralph M. Trüeb ${ }^{a}$ Laila El Shabrawi-Caelen ${ }^{b}$ Werner Kempf ${ }^{c}$ \\ ${ }^{a}$ Center for Dermatology and Hair Diseases Professor Trüeb, Zurich-Wallisellen, Switzerland; \\ ${ }^{b}$ Department of Dermatology, Medical University Graz, Graz, Austria; ${ }^{C}$ Kempf und Pfaltz Histologische \\ Diagnostik, Zurich, Switzerland
}

\section{Established Facts}

- Frontal fibrosing alopecia represents a condition with marginal scarring alopecia along the frontal and temporal hairline, which has been associated with further histopathologic and/or clinical evidence of lichen planopilaris.

- Recently, case reports and case series have given account of frontal fibrosing alopecia associated or overlapping with lupus erythematosus.

\section{Novel Insights}

- We present 2 cases of histopathologically proven cutaneous lupus erythematosus presenting as frontal fibrosing alopecia or frontal fibrosing alopecia-like cutaneous lupus erythematosus.

- In contrast to frontal fibrosing alopecia, frontal fibrosing alopecia-like cutaneous lupus erythematosus responded well to the respective treatment with oral hydroxychloroquine and/or intralesional triamcinolone acetonide.

\section{Keywords}

Frontal fibrosing alopecia . Cutaneous lupus erythematosus . Hydroxychloroquine $\cdot$ Intralesional triamcinolone acetonide

\begin{abstract}
Frontal fibrosing alopecia represents a peculiar condition with a quasi-symmetrical, marginal scarring alopecia along the frontal and temporal hairline. The condition has been associated with further histopathologic and/or clinical evidence of lichen planopilaris. Since its emergence with the
\end{abstract}

\section{KARGER}

(ㅇ 2017 S. Karger AG, Basel

E-Mail karger@karger.com

www.karger.com/sad original report of Kossard in 1994, frontal fibrosing alopecia has been recognized to be associated with a number of comorbidities, including lupus erythematosus. So far, respective case reports and case series have given account of frontal fibrosing alopecia with the histopathologic features of lichen planopilaris associated or overlapping with lupus erythematosus. In contrast, we present cases of histopathologically proven cutaneous lupus erythematosus presenting as frontal fibrosing alopecia with a good response to the respective treatment with oral hydroxychloroquine and/or intralesional triamcinolone acetonide.

(c) 2017 S. Karger AG, Basel 


\section{Introduction}

Frontal fibrosing alopecia (FFA) represents a peculiar condition with a quasi-symmetrical, marginal alopecia along the frontal and temporal hairline with scarring. Scalp biopsy specimens reveal histologic features that are usually indistinguishable from those seen in lichen planopilaris (LPP). Steven Kossard is credited with the original description of the condition in 1994 [1]. Eventually, Kossard interpreted FFA as a frontal variant of LPP [2]. Subsequent reports on oral lichen planus-like lesions [3] and lichen planus-type nail involvement [4] in patients with FFA provide further evidence that the condition may be closely related to lichen planus.

Since Kossard's original report in 1994, the number of cases of FFA has exploded exponentially worldwide, while its etiology has remained elusive. Familial cases of FFA point to the possible contribution of hereditary factors, possibly related to androgenetic alopecia [5-7], while recent questionnaire-based studies performed in patients with FFA suggest environmental factors, namely a possible association between FFA and the use of facial skin care products, particularly sunscreens $[8,9]$. The causality of this relationship remains to be confirmed since the study may have been biased through patient and question selection as well as confounding factors that were not included in the questionnaires.

Gaffney et al. [10] originally reported discoid lupus alopecia complicated by FFA on what the authors interpreted to be a background of androgenetic alopecia. While other authors have given account of further cases of FFA with the histopathologic features of LPP associated or overlapping with lupus erythematosus (LE) [11$14]$, herein we present 2 cases of histopathologically proven cutaneous LE presenting as FFA.

\section{Case Reports}

Case 1

A 64-year-old Caucasian woman complained of frontal and temporal hairline recession with patchy alopecia. Clinical inspection revealed a quasi-symmetrical recession of the respective hair line consistent with FFA, in association with patchy erythema. Extended examination disclosed associated small erythematous scaly lesions on the right helix and eyebrow region, suggestive of cutaneous LE (Fig. 1a). On dermoscopy, there was loss of follicular openings, together with enlarged branching vessels consistent with cutaneous LE [15] (Fig. 1b). Histopathological examination showed features compatible with cutaneous LE, such as vacuolar interface changes of the interfollicular epidermis (Fig. 1c), a dense superficial and deep perifollicular and perieccrine lymphohistiocytic in- filtrate with scattered plasma cells (Fig. 1d), increased mucin deposits identified with alcian blue staining, and a thickened PASpositive basement membrane zone. Direct immunofluorescence studies revealed a positive lupus band with granular depositions of IgM, IgG, and C3 along the basement membrane zone (Fig. 1e) and an elevated titer of SSA antibodies ( $28 \mathrm{U}$, normal: $<20 \mathrm{U})$, without further criteria for systemic lupus. The signs of cutaneous inflammation cleared with some regrowth of hair within 3 months under the administration of oral hydroxychloroquine ( $200 \mathrm{mg}$ b.i.d.) and topical clobetasol foam.

\section{Case 2}

A 65-year-old Caucasian woman was diagnosed and confirmed histopathologically with discoid LE (DLE) 13 years earlier and successfully treated with oral hydroxychloroquine. The medication was later withdrawn due to a drug eruption without recurrence of disease. After a long period of remission, she developed a patchy alopecia along the frontal and temporal hair line reminiscent of FFA, but with the histopathology of cutaneous LE, including positive direct immunofluorescence studies. Further clinical and laboratory workup did not reveal any signs of systemic LE, including a LE-specific serologic profile. Clinical examination showed atrophic alopecia with loss of sideburns, partial loss of eyebrows (Fig. 2a), and frontal alopecia (Fig. 2b). Dermoscopy of the central hair line revealed diversity of hair shaft diameters consistent with associated androgenetic alopecia. The patient was successfully treated with repeated intralesional triamcinolone acetonide 10 $\mathrm{mg} / \mathrm{mL}$ in combination with topical 5\% minoxidil, and 1,200 IU vitamin $\mathrm{E}$ and $500 \mathrm{mg}$ vitamin C daily (Fig. 2c).

\section{Discussion}

LE is a systemic autoimmune disorder associated with polyclonal B cell activation resulting in diverse patterns of autoantibody production, antibody-dependent cellular cytotoxicity, and a heterogeneous clinical expression constituting a spectrum extending from limited cutaneous disease to potentially life-threatening systemic disease. The characteristics of cutaneous LE have been well defined in terms of morphology, clinical, and laboratory criteria. Skin lesions have been classified into those that are histologically specific for LE and those that are not. The typical skin lesion of LE-specific disease on the scalp is DLE, with scalp involvement occurring in $60 \%$, and the only area involved in approximately $10 \%$ [16].

Typically, early DLE lesions of the scalp consist of scaling erythematous or violaceous papules which expand to form round or irregular shaped plaques with variable atrophy, follicular plugging, telangiectasia, and mottled areas of hyper- and hypopigmentation, while advanced lesions are scarring. Lesions of DLE are histologically characterized by vacuolar interface alteration involving the hair follicles and epidermis accompanied by a patchy su- 

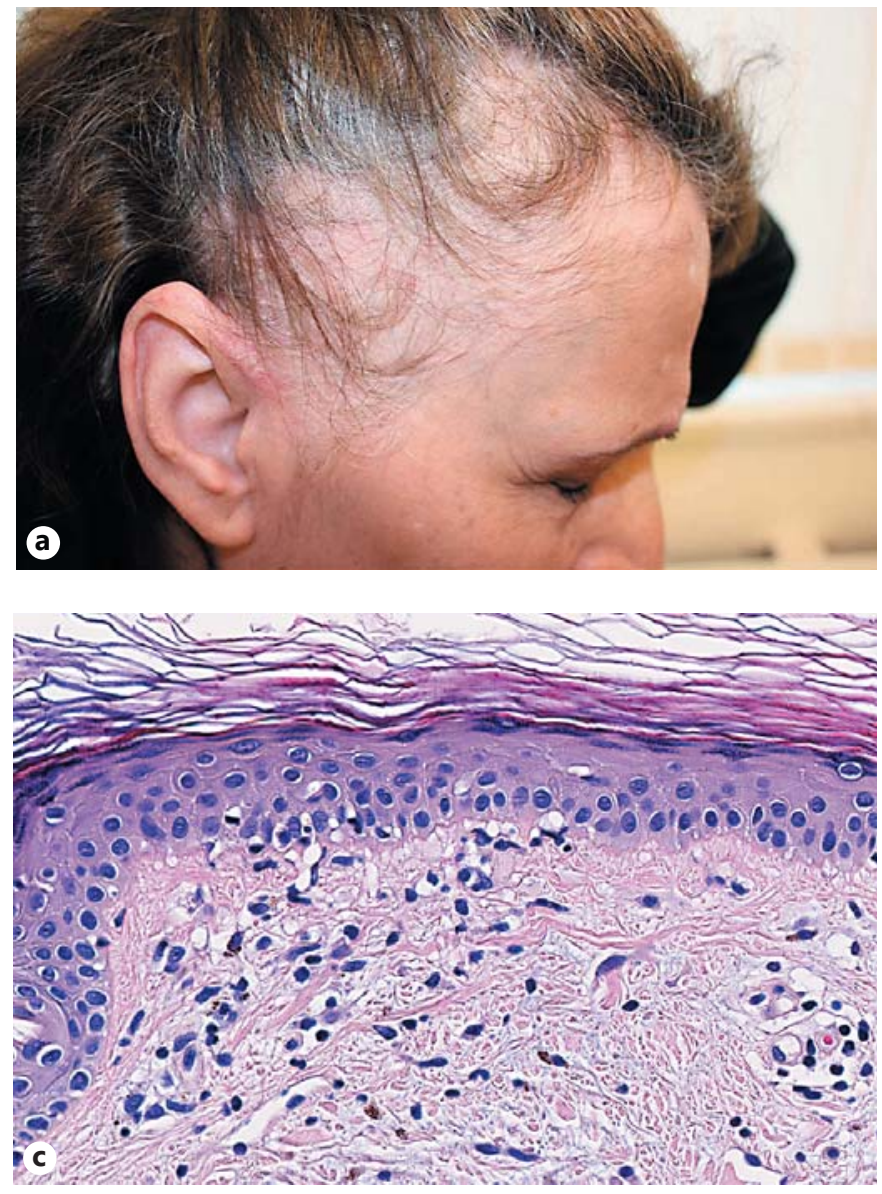

Fig. 1. a Frontal fibrosing alopecia-like lupus erythematosus associated with active skin lesions of lupus erythematosus. b Dermoscopy: loss of follicular openings and enlarged branching vessels. c Histopathology (H\&E): vacuolar interface dermatitis. d Dense superficial and deep perifollicular and perieccrine lymphohistiocytic infiltrates. e Direct immunofluorescence with a positive lesional lupus band test.

perficial and deep perivascular and periadnexal lymphocytic infiltrate. Laminated keratin fills dilated follicular ostia corresponding to the clinical follicular plugging. Minimal lymphocyte exocytosis into the basal layer keratinocytes of the follicular epithelium and interfollicular epidermis may be present, while apoptotic keratinocytes are often found both at the dermal-epidermal junction and

Cutaneous Lupus Erythematosus

Presenting as Frontal Fibrosing Alopecia
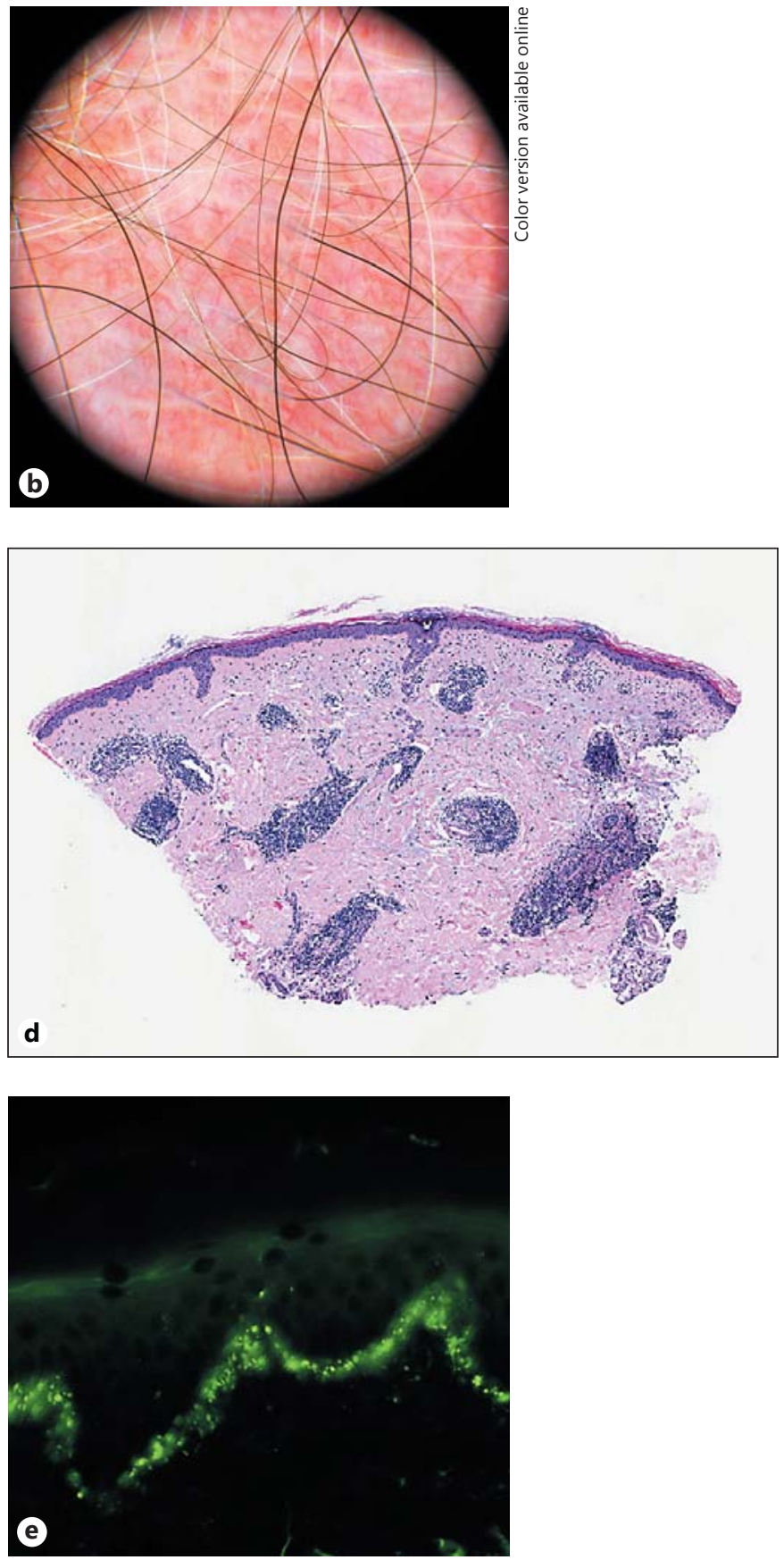

within the outer follicular epithelium. Basement membrane zone thickening initially consisting of a fine fibrillar reduplication of basal lamina recognizable only in special stains (PAS) progresses to form a broad homogenous band of eosinophilic basement membrane material. Increased dermal mucin is usually present both superficially and deep and is best detected in alcian blue-stained sec-

Skin Appendage Disord 2017;3:205-210 


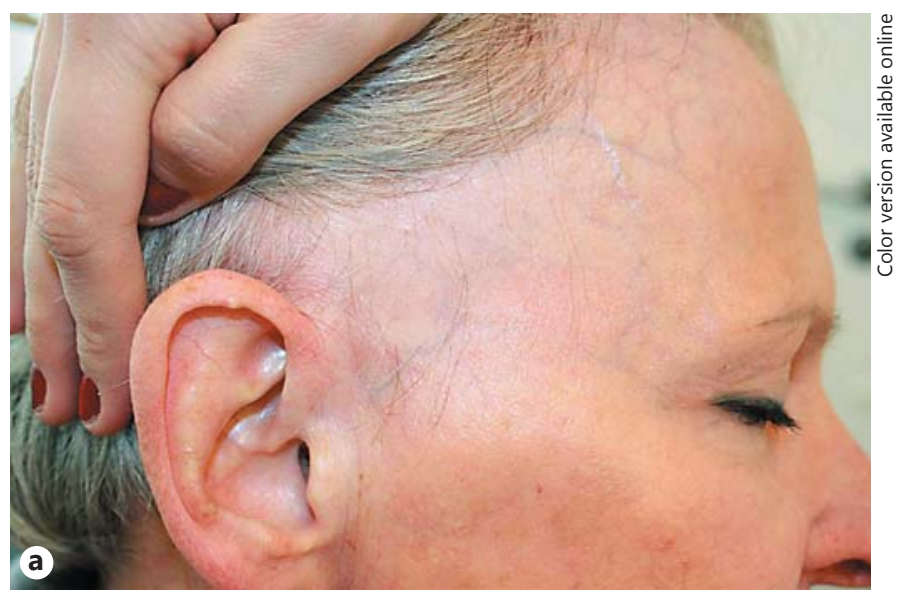

Fig. 2. a Temporal alopecia with loss of sideburns and loss of eyebrows. b, c Frontal hair loss before (b) and after (c) successful treatment.
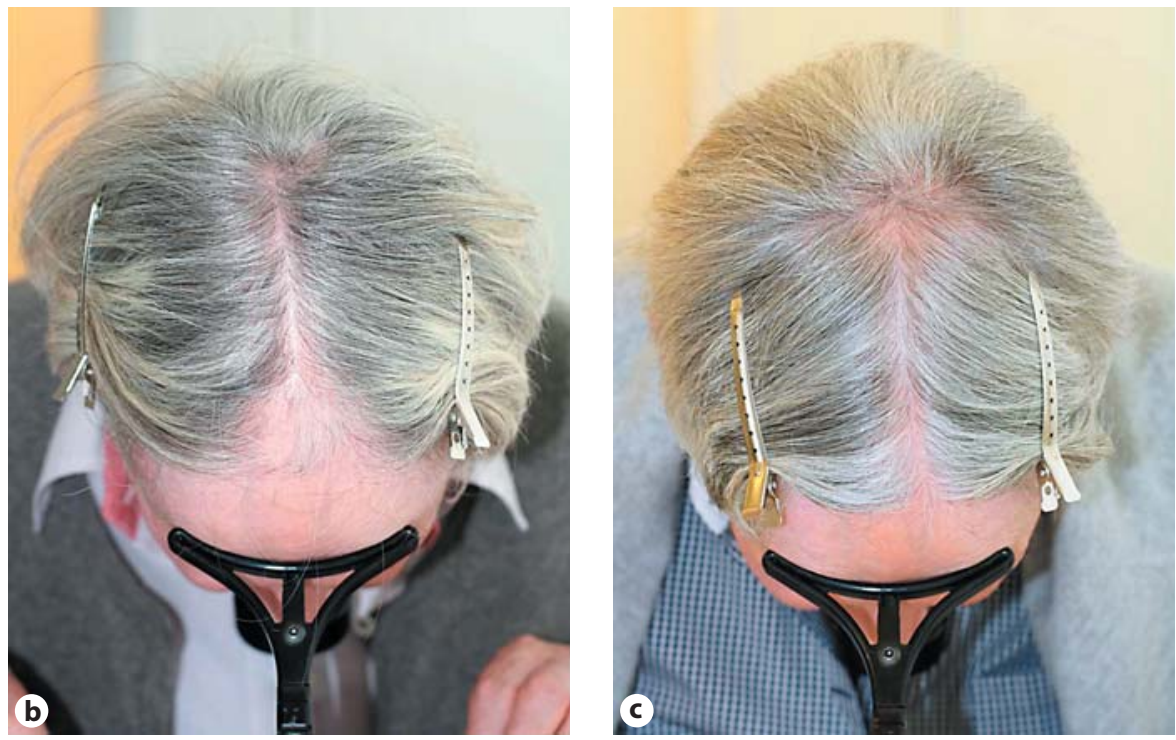

tions. Direct immunofluorescence studies demonstrate dense granular deposition of immunoglobulin (most commonly IgG, but IgM and IgA may also be present) and complement factor $\mathrm{C} 3$ at the dermal-epidermal junction in $76 \%$ of cases [17]. As with the other primary scarring alopecias, specifically LPP, there is no ideal treatment for the alopecias related to LE. Nevertheless, high-potency topical or intralesional corticosteroids as monotherapy or in combination with long-term antimalarial therapy, as indicated by the severity of the disease, may both halt the progression of DLE lesions of the scalp and speed the resolution of patchy alopecia that has not scarred yet. Because of the high risk of irreversible scarring alopecia, DLE of the scalp is best treated early and aggressively [18].

In the 2 cases presented, scalp biopsies of FFA-like alopecia fulfilled the histopathologic criteria of cutaneous
LE, including positive immunofluorescence studies. Up to date, 14 cases of FFA coexisting with LE have been reported [10-14]: Gaffney et al. [10] reported FFA in a female patient with preexisting DLE involving the head and neck. Khan et al. [11] described a male patient with FFA with histopathologic features of LPP and direct immunofluorescence studies consistent with a positive lupus band. Serologic testing confirmed the presence of antinuclear, anticardiolipin, and lupus anticoagulant antibodies; however, the patient was free of systemic symptoms. Wutte and El-Shabrawi-Caelen [12] observed a female patient with long-standing cutaneous LE of the face who developed FFA with the respective histopathology of LPP. The first series of 7 cases of coexistence of FFA and DLE of the scalp was provided by del Rei et al. [13]: all patients were females, none had systemic LE, other fea- 
Table 1. Proposal of classification of frontal fibrosing alopecia (FFA) (modified from [26])

- Postmenopausal FFA

- FFA in premenopausal women

- FFA in men

- Familial FFA

- FFA with associated skin, mucosal, or nail lichen planus

- FFA with associated autoimmune disease, e.g., lupus erythematosus, autoimmune thyroid disease

- Cutaneous lupus erythematosus presenting as FFA (FFA-like cutaneous lupus erythematosus)

tures associated with FFA were also seen, such as eyebrow loss, loss of body hair, and facial papules, and histopathologic features and direct immunofluorescence studies were again consistent with both LPP and DLE. Finally, Contin et al. [14] reported a case series of 4 female patients, 2 with DLE and 2 with systemic LE, with subsequent development of FFA that was nonresponsive to antimalarial therapy. Histopathologic and immunofluorescence studies were not included in the report.

Since its emergence in the 1990s, FFA has been recognized not only to present a more generalized than localized condition $[19,20]$ and to occur in a broader population than postmenopausal women, but also to be associated with comorbidities, notably of autoimmune origin, particularly autoimmune thyroid disease and LE [21-25]. In 2003, Trüeb [26] proposed in his textbook on hair dis- orders a classification of FFA into postmenopausal FFA as originally reported by Kossard, FFA in premenopausal women, FFA in men, FFA associated with other evidence of LPP, and FFA associated with other autoimmune disorders, including LE and autoimmune thyroid disease (Table 1).

In contrast, the cases presented herein do not represent FFA associated with LE, but rather LE presenting as FFA or FFA-like cutaneous LE. This has implications both for diagnostic and therapeutic strategies. We therefore strongly suggest performing a biopsy at the site of FFA in cases where associated skin lesions, suggestive dermoscopic findings, and/or the patient history may indicate LE, since our cases of FFA-like cutaneous LE responded well to the respective therapy with antimalarials and intralesional triamcinolone acetonide.

\section{Statement of Ethics}

The authors have no ethical conflicts to disclose. Patients' consent has been obtained.

\section{Disclosure Statement}

The authors have no disclosures to report. This publication results from the discussion of cases, respective dermatohistopathology, and pertinent literature on the occasion of Laila El ShabrawiCaelen's visit at the Center for Dematology and Hair Diseases Professor Trüeb March 13-17, 2017.

\section{References}

1 Kossard S: Postmenopausal frontal fibrosing alopecia: scarring alopecia in a pattern distribution. Arch Dermatol 1994;130:770-774.

2 Kossard S, Lee MS, Wilkinson B: Postmenopausal frontal fibrosing alopecia: a frontal variant of lichen planopilaris. J Am Acad Dermatol 1997;36:59-66.

3 Trüeb RM, Toricelli R: Lichen planopilaris unter dem Bild einer postmenopausalen frontalen fibrosierenden Alopezie (Kossard). Hautarzt 1998;49:388-391.

4 Macpherson M, Hohendorf-Ansari P, Trüeb RM: Nail involvement in frontal fibrosing alopecia. Int J Trichology 2015;7:64-66.

5 Junqueira Ribeiro Pereira AF, Vincenzi C, Tosti A: Frontal fibrosing alopecia in two sisters. Br J Dermatol 2010;162:1154-1155.

6 Dlova N, Goh CL, Tosti A: Familial frontal fibrosing alopecia. Br J Dermatol 2013;168: 220-222.

Cutaneous Lupus Erythematosus

Presenting as Frontal Fibrosing Alopecia
7 Navarro-Belmonte MR, Navarro-López V, Ramírez-Boscà A, Martínez-Andrés MA, Molina-Gil C, González-Nebreda M, AsínLlorca M: Case series of familial frontal fibrosing alopecia and a review of the literature. J Cosmet Dermatol 2015;14:64-69.

8 Aldoori N, Dobson K, Holden CR, et al: Frontal fibrosing alopecia: possible association with leave-on facial skin care products and sunscreens; a questionnaire study. Br J Dermatol 2016;175:762-767.

9 Debroy-Kidambi A, Dobson K, Holmes S, et al: Frontal fibrosing alopecia in men - an association with facial moisturisers and sunscreens. Br J Dermatol 2017, DOI: 10.1111/ bjd.15311.

10 Gaffney DC, Sinclair RD, Yong-Gee S: Discoid lupus alopecia complicated by frontal fibrosing alopecia on a background of androgenetic alopecia. Br J Dermatol 2013;169:217218.
11 Khan S, Fenton DA, Stefanato CM: Frontal fibrosing alopecia and lupus overlap in a man: guilt by association? Int J Trichology 2013;5: 217-219.

12 Wutte N, El-Shabrawi-Caelen L: Frontal hair loss and facial skin changes. J Dtsch Dermatol Ges 2015;13:1040-1043.

13 del Rei M, Pirmez R, Sodré CT, Tosti A: Coexistence of frontal fibrosing alopecia and discoid lupus erythematosus of the scalp in $7 \mathrm{pa}$ tients: just a coincidence? J Eur Acad Dermatol Venereol 2016;30:151-153.

14 Contin LA, Martins da Costa Marques ER, Noriega L: Frontal fibrosing alopecia coexisting with lupus erythematosus: poor response to hydroxychloroquine. Skin Appendage Disord 2017;2:162-165.

15 Lallas A, Apalla Z, Lefaki I, et al: Dermoscopy of discoid lupus erythematosus. Br J Dermatol 2013; 168:284-288. 
16 Prystowsky SD, Herndon JH, Gilliam JN: Chronic cutaneous lupus erythematosus (DLE): a clinical and laboratory investigation of 80 patients. Medicine 1976;55:183-191.

17 Trachsler S, Trueb RM: Value of direct immunofluorescence for differential diagnosis of cicatricial alopecia. Dermatology 2005;211: 98-102.

18 Trüeb RM: Involvement of scalp and nails in lupus erythematosus. Lupus 2010;19:10781086.
19 Armenores P, Shirato K, Reid C, Sidhu S: Frontal fibrosing alopecia associated with generalized hair loss. Australas J Dermatol 2010;51:183-185.

20 Chew AL, Bashir SJ, Wain EM, et al: Expanding the spectrum of frontal fibrosing alopecia: a unifying concept. J Am Acad Dermatol 2010;63:653-660.

21 Samrao A, Chew AL, Price V: Frontal fibrosing alopecia: a clinical review of 36 patients. Br J Dermatol 2010;163:1296-1300.

22 MacDonald A, Clark C, Holmes S: Frontal fibrosing alopecia: a review of 60 cases. J Am Acad Dermatol 2012;67:955-961.

23 Ladizinski B, Bazakas A, Selim MA, Olsen EA: Frontal fibrosing alopecia: a retrospective review of 19 patients seen at Duke University. J Am Acad Dermatol 2013;68:749-755.
24 Banka N, Mubki T, Bunagan MJ, McElwee K, Shapiro J: Frontal fibrosing alopecia: a retrospective clinical review of 62 patients with treatment outcome and long-term follow-up. Int J Dermatol 2014;53:1324-1330.

25 Vañó-Galván S, Molina-Ruiz AM, SerranoFalcón C, et al: Frontal fibrosing alopecia: a multicenter review of 355 patients. J Am Acad Dermatol 2014;70:670-678.

26 Trüeb RM: Haare. Praxis der Trichologie. Darmstadt, Steinkopff, 2003, pp 291. 